\title{
Comparison of In Situ Micromechanical Strain-Rate Sensitivity Measurement Techniques
}

\author{
JURI WEHRS, ${ }^{1,4}$ GAURAV MOHANTY, ${ }^{1}$ GAYLORD GUILLONNEAU, ${ }^{1}$ \\ AIDAN A. TAYLOR,${ }^{1}$ XAVIER MAEDER,${ }^{1}$ DAMIAN FREY, ${ }^{1}$ \\ LAETITIA PHILIPPE ${ }^{1}$ STEFANO MISCHLER ${ }^{2}$ \\ JEFFREY M. WHEELER, ${ }^{1,3}$ and JOHANN MICHLER ${ }^{1}$
}

\begin{abstract}
1.-Laboratory for Mechanics of Materials and Nanostructures, Empa, Swiss Federal Laboratories for Materials Science and Technology, 3602 Thun, Switzerland. 2.-Tribology and Interface Chemistry Group, Materials Institute, École Polytechnique Fédérale de Lausanne, 1015 Lausanne, Switzerland. 3.-Laboratory for Nanometallurgy, Department of Materials Science, ETH Zürich, 8093 Zurich, Switzerland. 4.—e-mail: Juri.Wehrs@empa.ch
\end{abstract}

Strain-rate sensitivity (SRS) measurements using transient small-scale techniques are becoming increasingly popular for investigating nanostructured films and microcomponents since they can provide fundamental insights into the plastic deformation mechanisms within small volumes of material. Previously, researchers have typically used either nanoindentation or microcompression strain-rate jump tests on a variety of nanostructured materials and compared the resultant values with bulk compression data as a reference. However, no systematic comparison of the different transient micromechanical techniques has been performed on the same material to establish their relative merits or the consistency of their results. In this study, the SRS of nanocrystalline nickel is investigated using three independent, in situ, transient experimental techniques: miniature tension, nanoindentation, and micropillar compression. The obtained SRS exponents $m$ measured by all techniques were found to be in good agreement, and the resulting apparent activation volume $V_{\text {app }}$ of approximately $10 b^{3}$ is consistent with grain boundary diffusion processes and dislocation glide-based plasticity.

\section{INTRODUCTION}

Compared with conventional polycrystalline facecentered cubic (fcc) metals, nanocrystalline metals provide excellent mechanical properties, ${ }^{1-3}$ which makes these materials very attractive for a variety of applications. The key parameter that determines the mechanical properties in these materials is their extremely fine grain size, which is the cause of both their greatly improved strength and their highly time-dependent plastic behavior. As the grain size is reduced, the relative amount of volume occupied by grain boundaries is increased. For example, the grain boundaries occupy volume fractions as large as $\sim 5 \%$ to $\sim 10 \%$ for grain sizes of $50 \mathrm{~nm} .^{4,5}$ This strengthens the influence of grain boundary-mediated processes like grain boundary sliding, grain boundary migration, ${ }^{6}$ and dislocation nucleation. ${ }^{3,7}$ Consequently, both thermally activated and inelas- tic, stress-driven deformation processes can be simultaneously operative in these materials. ${ }^{8,9}$ All of these mechanisms contribute toward the increased strainrate sensitivity (SRS) of nanocrystalline metals.

With the exception of materials made by severe plastic deformation techniques, ${ }^{10}$ the majority of nanostructured materials are not available in bulk quantities. Production techniques, such as electrodeposition or inert gas condensation, generally yield small quantities of thin films. This reduces the range of suitable conventional mechanical testing techniques that are scalable and sensitive enough to probe small quantities of materials in a reproducible manner. As a necessity, standard bulk tests have to be adapted to the desired length scales and specific sample geometries. In particular, variable strainrate tests have to be modified for miniaturized specimens to asses SRS and gain fundamental insights into the rate-controlling deformation mechanism(s). 
The two main variations in SRS measurement techniques are constant strain rate (CSR) testing, where multiple samples are tested at different constant strain rates, and strain-rate jump (SRJ) testing, where the applied strain rate is abruptly changed during a testing on a single sample. Because tension testing is perhaps the most fundamental of all mechanical testing techniques, both variations have traditionally been used to study the SRS of metals. Consequently, tensile testing has been performed both ex situ and in situ on various nanostructured materials using monotonic, CSR techniques on different length scales from bulk to the nanoscale. ${ }^{11-15}$ Wang et al. ${ }^{16}$ performed transient, strain-rate jump (SRJ) tests as a function of temperature on nanocrystalline nickel specimens with a thickness of $150 \mu \mathrm{m}$ and a grain size of $15 \mathrm{~nm}$. Jonnalagadda et al. reduced the specimen size further to microtensile samples of gold ${ }^{17}$ and platinum ${ }^{18}$ with gauge thicknesses of $<2 \mu \mathrm{m}$. On the lower end of the length scale, Gianola et al. ${ }^{19}$ performed SRJ tests on free-standing aluminum films with thicknesses as small as $150 \mathrm{~nm}$.

However, the production of microtensile or nanotensile specimens for mechanical testing can be a complicated matter. This causes many researchers to turn to nanoindentation ${ }^{20-22}$ as an attractive alternative to tensile testing. Primarily, these efforts have focused on constant strain-rate techniques, but Pan et al. ${ }^{23}$ and Maier et al. ${ }^{24}$ recently demonstrated methods to perform SRJ nanoindentation tests, which allow SRS and activation volumes to be extracted with greater speed, consistency, and decreased likelihood of error from creep or drift. The method has now been extended to elevated temperatures ${ }^{25,26}$ allowing the extraction of activation energy as well.

In parallel to these efforts, the advent of focused ion beam (FIB) machining techniques enables fabrication of uniaxial loading geometries that made compression $^{27}$ and tension ${ }^{28}$ tests possible at the microscale and nanoscale. These techniques allow the uniaxial and/or anisotropic behavior of samples to be measured directly rather than inferring them from the complicated stress and strain gradients that develop in indentation. Wheeler et al. ${ }^{29}$ first demonstrated the application of reversible, transient strain-rate jumps during microcompression testing. This work showed good agreement between SRS values determined by both CSR and SRJ measurements performed in situ in a scanning electron microscope (SEM) on brittle GaN prisms. Independently, at nearly the same time, Carpenter et al. ${ }^{30}$ used increasing rate jumps to measure the SRS of metallic multilayer micropillars at room temperature. Mohanty et al. ${ }^{31}$ conducted the first systematic micropillar SRJ tests as a function of temperature. This was performed in situ in the SEM on nanocrystalline nickel micropillars with an average grain size of $27 \mathrm{~nm}$. The SRS exponent was observed to increase from 0.015 to 0.028 from $25^{\circ} \mathrm{C}$ to $100^{\circ} \mathrm{C}$. This resulted in a decrease of apparent activation volume from $\sim 21 b^{3}$ to $\sim 11 b^{3}$. The extracted parameters were in broad agreement with the existing literature, and the apparent activation energy suggested grain boundary diffusion mechanisms to be rate controlling.

Although a variety of metallic and semiconductor materials has now been investigated by various strain-rate jump test methodologies, currently, no systematic comparison of the different micromechanical techniques has yet been performed on the same material to establish their relative merits or the consistency of their results. The current study aims to examine the comparability of three different in situ, small-scale mechanical testing methods using relatively well-established techniques like tensile testing against techniques that are of more recent origin, like nanoindentation and microcompression. To achieve maximum comparability of results, all three techniques (miniature tension, nanoindentation, and micropillar compression) were used on the same electrodeposited nanocrystalline nickel specimen. The resulting SRS exponents and apparent activation volumes from these different methods will be compared and discussed.

\section{EXPERIMENTAL PROCEDURE}

\section{Material}

Nominally pure, nanocrystalline nickel was produced by means of a proprietary electrodeposition process on a silicon wafer. An ultraviolet LIGA $^{32}$ method was used to produce individual free-standing, dogbone-shaped microtensile bars, as shown in the inset of Fig. 1. The samples had a gauge length of $3.0 \mathrm{~mm}$, gauge width of $0.15 \mathrm{~mm}$, and thickness of $0.25 \mathrm{~mm}$. The top surface of the deposited

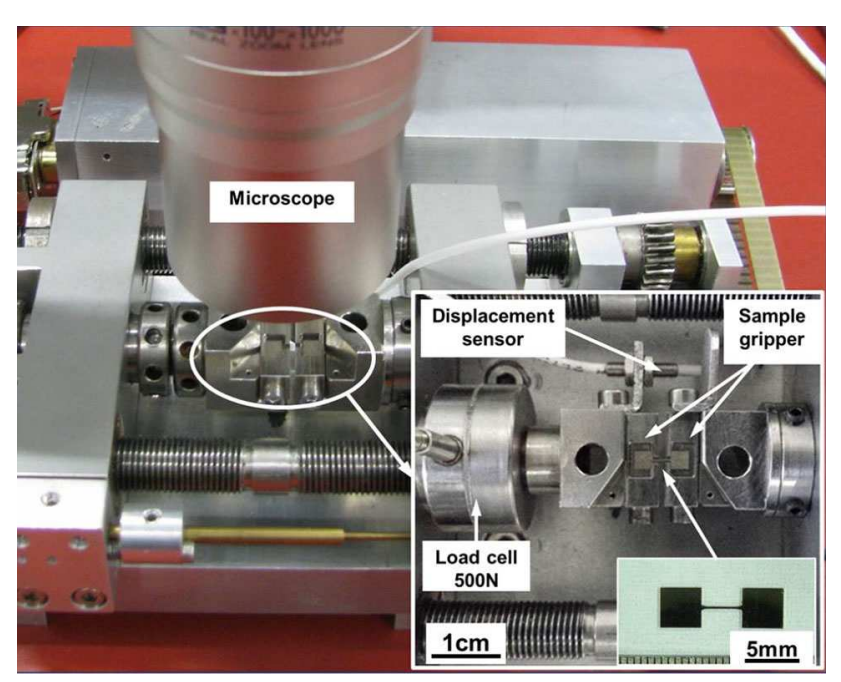

Fig. 1. Adapted miniaturized tensile stage with 500-N load cell, displacement sensor, and hard metal grippers. A digital microscope fixed perpendicular to the specimen is used for strain measurement. The inset in the right figure shows a nanocrystalline nickel microtensile bar (LIGA). 
samples was lightly polished using standard metallographic techniques to reduce surface roughness.

As a result of the bath chemistry (components and additives), electrodeposits are generally less pure than nanocrystalline materials produced by alternative routes such as inert gas condensation or sputtering. ${ }^{1}$ Some additives, like refractory metals, are intended to refine and thermally stabilize the nanostructure and enhance the strength of the deposited material. In the case of nickel, refractory metals like tungsten or cobalt are commonly used for this purpose. ${ }^{33-35}$ On the other hand, light interstitial elements like carbon or boron ${ }^{36-38}$ can also have a major influence on the mechanical properties of the material. Some impurities, such as sulfur, can cause embrittlement by segregating to and weakening the grain boundaries upon annealing. ${ }^{39}$ To determine the actual composition of the electrodeposited material, glow discharge optical emission spectroscopy (GD-OES) was performed on the samples using a JY $5000 \mathrm{RF}$ instrument (HORIBA Jobin-Yvon SAS, Longjumeau Cedex, France). The composition was determined to be $\mathrm{Ni}=99.81$, $\mathrm{S}=0.06, \mathrm{C}=0.04$, remainder $=0.09(\operatorname{mass} \%)$.

To determine the electrodeposited material's texture and crystallite size, x-ray diffraction (XRD) measurements were performed using a Discover D8 diffractometer (Bruker $\mathrm{GmbH}$, Karlsruhe, Germany) with a $\mathrm{Cu}-\mathrm{K} \alpha$ source operated at $40 \mathrm{kV} /$ $40 \mathrm{~mA}$. The texture of the samples was estimated from the (111), (200), (220), and (311) peaks from the Bragg-Brentano theta-2theta diffractograms, using the Harris method. ${ }^{40}$ The results indicate a strong (111) texture. The average crystallite size was estimated with the Scherrer formula ${ }^{41,42}$ from the full width at half maximum of the (111) peak, which yielded a mean value of $d_{\mathrm{XRD}, \mathrm{Ni}} \cong 15 \mathrm{~nm}$. However, this estimation is only a lower boundary estimate because some parameters, such as the presence of twins, crystal defects, and residual stresses, can also contribute to the peak broadening. ${ }^{31}$

For additional characterization of the material's microstructure and grain size, transmission electron microscopy (TEM) was performed. TEM lamellae of the deposit were manufactured by the FIB lift-out technique ${ }^{43}$ using a Vela dual-beam FIB (TESCAN s.r.o., Brno, Czech Republic). The surface of the deposit was coated using a gas injection system with $2 \mu \mathrm{m}$ of platinum prior to lift out to provide a protective layer to limit the extent of any ion beam damage. Final polishing of the lamellae was performed using a $5 \mathrm{kV}$ ion beam at $1^{\circ}$ incidence to the lamellae surfaces. A JEM-2200FS TEM (JEOL $\mathrm{GmbH}$, München, Germany) was used to image the sample (Fig. 2) in scanning mode (scanning transmission electron microscope) at $200 \mathrm{kV}$ and $0.7 \mathrm{~nm}$ spot size. The material's nanostructure displayed a homogeneous grain size distribution with an average grain size of $26 \pm 10 \mathrm{~nm}$, calculated for more than 50 grains applying grain area analysis using

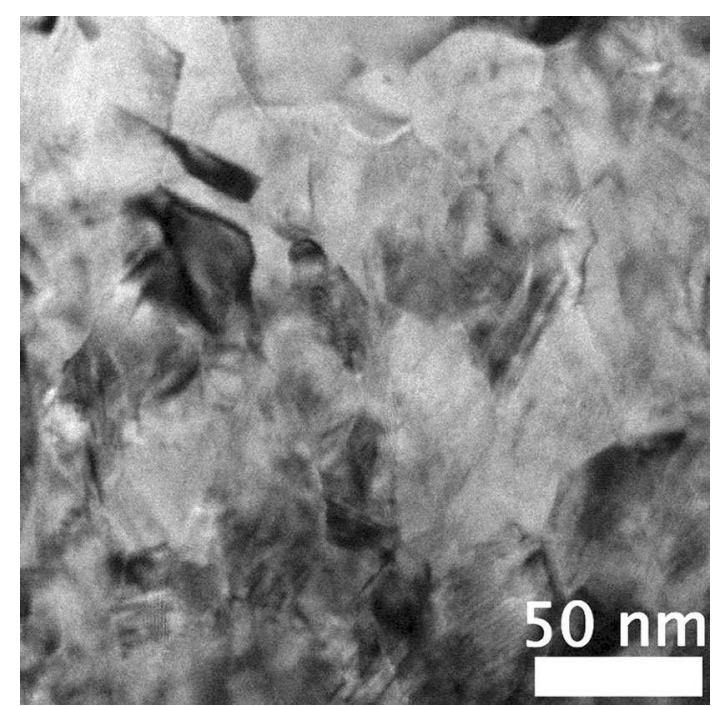

Fig. 2. Bright-field TEM image of nanocrystalline nickel. The grain size is in the order of $26 \mathrm{~nm}$.

the ImageJ software package (National Institutes of Health, Bethesda, Maryland).

To enhance the comparability of results and avoid any sample to sample variations in composition and/ or microstructure obtained from a single electrodeposited wafer, the full series of nanoindentation and micropillar compression tests in this work were performed on the undeformed ends of one of the dogbone tensile specimens postmortem.

\section{Miniature Tensile Experiments}

Displacement-controlled miniature tensile experiments were conducted to investigate the bulk mechanical behavior of nanocrystalline nickel. A commercially available microtensile stage (Kammrath \& Weiss GmbH, Dortmund, Germany) with a nominal load capacity of $25 \mathrm{kN}$ was previously adapted $^{44}$ for testing LIGA samples as shown in Fig. 1 . The spindle-driven system is equipped with a compact load cell (Measurement Specialties Ltd., Hampton, VA) with a noise floor of $0.03 \mathrm{~N}$ and a maximum load of $500 \mathrm{~N}$. An inductive displacement sensor is directly attached to the sample grip support. However, this sensor is only used as a reference signal for applying desired displacement rates during the experiment. To avoid any error in displacement arising from the compliance of the setup or deformation outside of the gauge section, the actual displacement of the sample gauge section was measured in situ by optical flow measurement from high-definition video acquired using a VHX500F digital microscope (KEYENCE International SA, Mechelen, Belgium) mounted perpendicular to the specimen. A Lucas and Kanade algorithm ${ }^{45}$ was implemented in LabVIEW-based software (National Instruments Corporation, Austin, TX) to measure the time-dependent displacement between selected points on the gauge section. 
Tensile tests were performed using monotonic, CSR, and transient SRJ techniques. CSR tensile tests were conducted on a minimum of three samples of nanocrystalline nickel and maraging steel at a strain rate of $\dot{\varepsilon}_{\text {CSR tension }}=4 \times 10^{-4} \mathrm{~s}^{-1}$ until specimen failure. SRJ tests were performed on five nanocrystalline-nickel samples where the strain rate was varied over two orders of magnitude from $\dot{\varepsilon}_{\text {SRJ,tension }}=8.8 \times 10^{-5} \mathrm{~s}^{-1}$ to $1.2 \times 10^{-3} \mathrm{~s}^{-1}$.

\section{Nanoindentation Testing}

In situ nanoindentation was performed at room temperature using a SEM Indenter system (Alemnis $\mathrm{GmbH}$, Thun, Switzerland) inside a DSM962 SEM (Carl Zeiss Microscopy GmbH, Oberkochen, Germany). Details of the system have been published in earlier works. ${ }^{46,47}$ Because the system is intrinsically displacement controlled using a highly responsive piezoelectric actuator-rather than using a feedback loop to attempt to maintain a nearly constant displacement rate with a force actuator-strain rates can be varied back and forth over three orders of magnitude $\left(10^{-2} \mathrm{~s}^{-1}\right.$ to $10^{-5} \mathrm{~s}^{-1}$ ) in a single experiment with a high degree of accuracy without needing a high-speed feedback loop.

One main advantage of in situ testing is the ability to constantly observe the sample and indenter during all stages of the experiment. This significantly increases the quality of the data by ensuring that no debris, specimen defects, or misalignments are influencing the experiment. Furthermore, observation of specimen deformation can provide both qualitative (deformation mechanisms: shear bands, slip steps, pile-ups, etc.) and quantitative (optical strain measurement) information during testing.

To ensure that the representative strain level was comparable with both the tension and the microcompression testing, nanoindentation testing was performed using a calibrated ${ }^{48}$ diamond Berkovich indenter, $\varepsilon_{\mathrm{R}}=8 \%$, rather than a Cube Corner indenter $\varepsilon_{\mathrm{R}}=22 \%,{ }^{24}$ which would have provided superior in situ observation. A limitation of previous work $^{25}$ using this system to characterize SRS using indentation was the lack of continuous stiffness measurement (CSM, which is also alternatively referred to as sinus mode or dynamic testing) capability $^{49}$ to measure the hardness and modulus as a function of depth during the jump segments. However, in collaboration with Alemnis $\mathrm{GmbH}$, this has now been developed. An oscillation with constant voltage amplitude was superimposed on the excitation of the displacement piezoelectric stack. This resulted in a displacement amplitude of approximately $5 \mathrm{~nm}$ at a frequency of $25 \mathrm{~Hz}$. The indentation modulus and the hardness were extracted from each sinus segment using the Oliver and Pharr method. ${ }^{50}$ Strain-rate jumps were incorporated during each indentation cycle using the method described by Maier et al. ${ }^{24}$ The indentation strain rate, defined as the ratio of the loading rate $\dot{P}$ to the current load $P$ has been shown ${ }^{51}$ to be proportional to the strain rate when the hardness $H$ is constant with depth $(\dot{H} / H=0)$ at depths above that where an indentation size effect is observed:

$$
\dot{\varepsilon}=\frac{\dot{h}}{h}=\frac{1}{2}\left(\frac{\dot{P}}{P}-\frac{\dot{H}}{H}\right) \approx \frac{1}{2} \frac{\dot{P}}{P}
$$

The indentation strain rates were varied from $\dot{\varepsilon}_{\text {SRJ,indentation }}=2 \times 10^{-2} \mathrm{~s}^{-1}$ to $5 \times 10^{-5} \mathrm{~s}^{-1}$ during the loading cycle. The hardness was measured for depths greater than $500 \mathrm{~nm}$ to avoid any size effects. ${ }^{24}$ During unloading, the strain rate was kept constant at $\dot{\varepsilon}_{\text {SRJ,indentation,unloading }}=2 \times 10^{-3} \mathrm{~s}^{-1}$ and the thermal drift was corrected by applying a constant load hold segment at $95 \%$ of unloading. A relatively high data acquisition rate of $2500 \mathrm{~Hz}$ was used to capture the sinus oscillations with sufficient resolution at the higher strain rates. The modulus and hardness results obtained were averaged more than five sinus oscillation cycles. More than 10 indentations were performed with different strain rate jump profiles (i.e., varying the order of the strain-rate magnitudes occurring at different indentation depth segments) to illustrate the reversibility of the jumps ${ }^{24}$ and ensure that the hardness is a function of indentation strain rate and independent of indentation depth.

\section{Microcompression Testing}

Micropillars with a nominal diameter of $3.5 \mu \mathrm{m}$ and an aspect ratio of 2.5 were machined using a TESCAN Lyra gallium FIB system at $30 \mathrm{kV}$. A three-step milling procedure was chosen, starting with a coarse milling at $4.2 \mathrm{nA}$ beam current. After a fine milling step with $1.2 \mathrm{nA}$, the final polishing was done at $0.2 \mathrm{nA}$. For further comparison, micropillars were also fabricated on a 123-oriented single crystal of pure nickel, acquired from Goodfellow Ltd. (Cambridge, U.K).

Microcompression testing was performed using the same SEM Indenter system described in the preceding section. However, a $5-\mu \mathrm{m}$ diameter diamond flat-punch indenter tip was used instead of a Berkovich indenter. CSR tests were conducted with a strain rate of $\dot{\varepsilon}_{\mathrm{CSR} \text {,compression }}=7 \times 10^{-4} \mathrm{~s}^{-1}$ to a total strain of $11 \%$. The applied strain rates were varied from $\dot{\varepsilon}_{\text {SRJ,compression }}=2 \times 10^{-2} \mathrm{~s}^{-1}$ to $4 \times 10^{-5} \mathrm{~s}^{-1}$ on each micropillar. Similar to the nanoindentation experiments, the strain rate for the last segment was kept the same as for the first segment to demonstrate reversibility. Additionally, the order of the strain rate jumps was changed for a few tests to ensure that the strain hardening of the material did not influence the test results. A minimum of three pillars were compressed on both the 


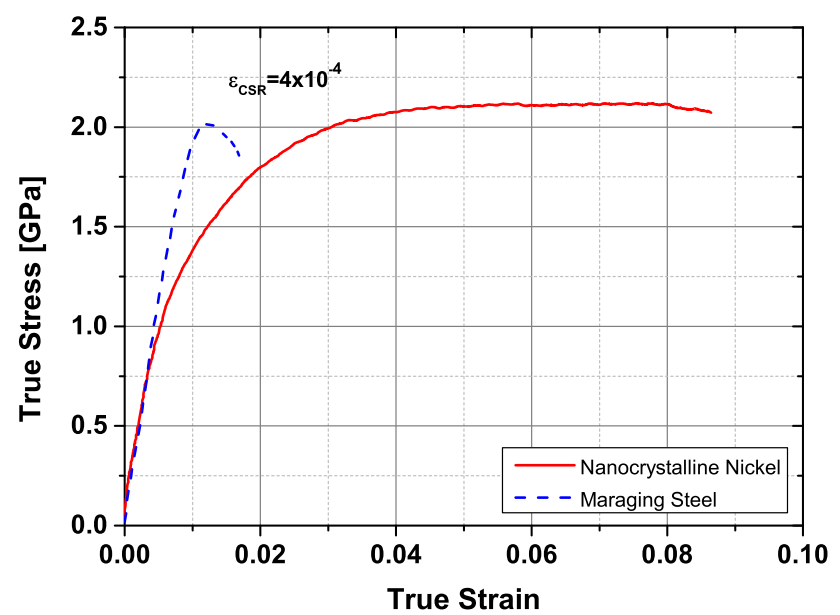

Fig. 3. Tensile stress-strain curves of nanocrystalline nickel and polycrystalline maraging steel at CSR.

nanocrystalline nickel and the 123-oriented singlecrystal nickel samples.

\section{RESULTS AND DISCUSSION}

\section{Miniature Tension}

Tensile testing is perhaps the most standard technique for measuring mechanical properties and, as such, provides a good baseline against which to compare the results of more novel techniques. Figure 3 shows representative true stress-strain curves obtained from CSR tension tests performed at a strain rate of $\dot{\varepsilon}_{\text {CSR.tension }}=4 \times 10^{-4} \mathrm{~s}^{-1}$. The results from nanocrystalline-nickel samples indicate a Young's modulus of $E_{\mathrm{ncNi}}=180 \pm 12 \mathrm{GPa}$ (where $\mathrm{nc}=$ nanocrystalline $)$ and yield strength at $1 \%$ offset of $\sigma_{y, \mathrm{ncNi}}=1.8 \pm 0.2 \mathrm{GPa}$. Significant work hardening and ductility is also observed.

These results are difficult to compare directly with other researchers' results in the literature because it is known that the mechanical properties of nanocrystalline materials depend heavily on factors like grain size, texture, impurities, and processing parameters. To ensure the accuracy of the applied testing apparatus and method, reference measurements on a well-characterized bulk material were also conducted. Miniature tensile bars were made using electrical discharge machining (EDM) from rolled maraging steel sheets of similar thickness to the nanocrystalline-nickel LIGA samples. Tensile results from these samples yielded a Young's modulus $E_{\text {maragingsteel }}=205 \pm 10 \mathrm{GPa}$ and $\mathrm{UTS}_{\text {maragingsteel }}=2.0 \pm 0.12 \mathrm{GPa}$ (where UTS $=$ ultimate tensile strength). These results match well with the bulk properties given by the manufacturer $\left(E_{\text {maragingsteel,ref }}=208 \mathrm{GPa}\right.$ and $\mathrm{UTS}_{\text {maragingsteel,ref }}=$ $2.05 \mathrm{GPa}$ ). This suggests that the applied testing and data evaluation method is valid and delivers reasonable results for the nanocrystalline nickel.

Most studies on the SRS of nanocrystalline metals under tensile loading have concentrated on series of monotonic, CSR tests in which individual specimens are strained at a single strain rate for the entire duration of the test. This is repeated for several different strain rates with a different specimen for each test. Transient tests, in which the strain rate changes during the test, provide the advantage of extracting rate sensitivity from a single test by varying the strain rate and monitoring the materials response in flow stress. ${ }^{52}$ This allows measurement of the rate sensitive flow behavior of a constant microstructure and removes the influence of any microstructural variation between the samples. However, the ductility of nanocrystalline materials is generally limited to a few percentage strain before plastic instability (nonuniform deformation such as necking) occurs, and this generally limits the feasibility of transient testing. Furthermore, the lack of strain hardenability, due to the small grain size, reduces the magnitude of the plastic regime further. The location of this plastic instability is generally attributed to defects (e.g., pores or foreign species), which lead to localized stress concentrations. The nature and quantity of these defects can vary heavily with the production method of the samples. After onset of plastic instability, which is described by the Considére-criterion: ${ }^{53}$

$$
\left(\frac{\partial \sigma}{\partial \varepsilon}\right)_{\dot{\varepsilon}} \leq \sigma
$$

The stress state is no longer purely uniaxial, so experimental observations will differ from the theory. The inset in Fig. 4 shows a tensile bar during necking, which is first observed at approximately $8.2 \%$ strain. In a well-designed transient test, all of the rate jumps will be completed well before the onset of necking. Therefore, the number of possible strain rates (in the strain-rate range of interest) that can be accommodated within one test using this apparatus was limited to three. Figure 4 displays the sequence of SRJ on a representative stress strain curve. The initial strain rate was set to $\dot{\varepsilon}_{\mathrm{SRJ}, 1}=4 \times 10^{-4} \mathrm{~s}^{-1}$ until well into the plastic regime. Reducing the strain rate to $\dot{\varepsilon}_{\mathrm{SRJ}, 2}=8.8 \times 10^{-5} \mathrm{~s}^{-1}$ showed an immediate reduction in flow stress. Increasing the strain rate back to $\dot{\varepsilon}_{\mathrm{SRJ}, 1}$ resulted in an increase in flow stress back to the previous level as predicted by the fitted curve. This shows the flow stress transient testing to be fully reversible, indicating a mostly constant microstructure. This same reversibility was observed after increasing the strain rate further by another order of magnitude to $\dot{\varepsilon}_{\mathrm{SRJ}, 3}=1.2 \times 10^{-3} \mathrm{~s}^{-1}$. After each SRJ, the flow stress level was observed to quickly reach a steady state.

\section{Nanoindentation}

A representative load-displacement curve from nanoindentation SRJ experiment is shown in Fig. 5. The individual SRJ segments are marked with their 


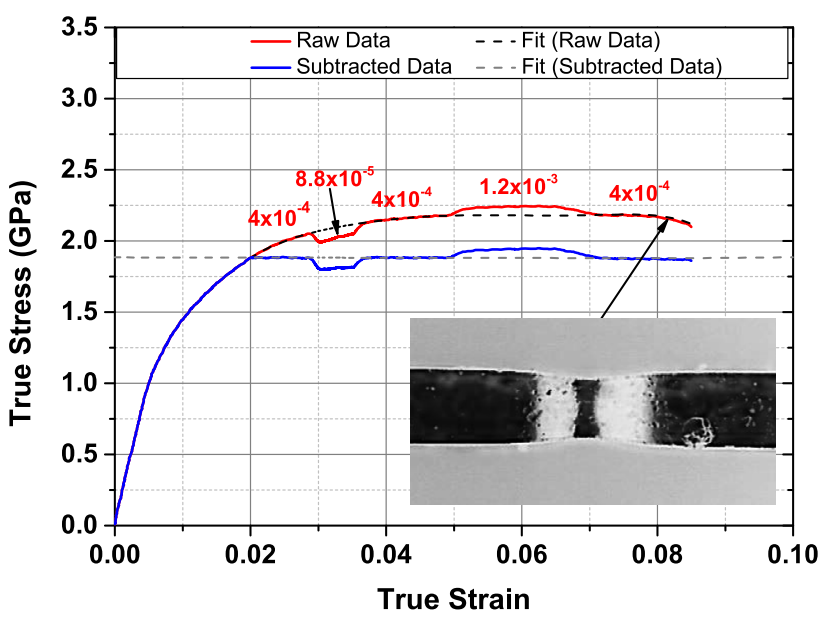

Fig. 4. Tensile stress-strain curves for SRJ tests on nanocrystalline nickel. The red curve shows the raw data, whereas the black dashed curve represents a fit through the initial and final segment of the red curve. To account for strain hardening, the fit was subtracted from the red curve and shifted in stress axis to result in zero slope (blue curve) (Color figure online).

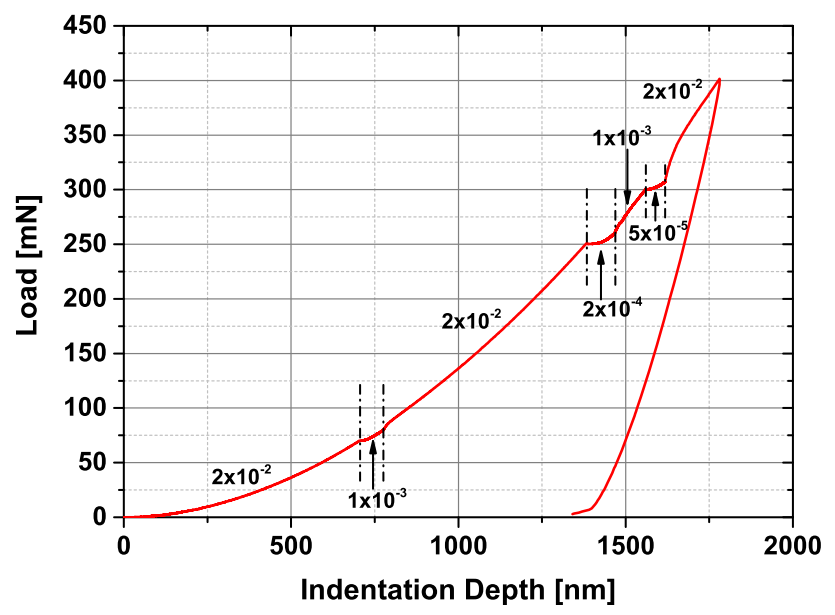

Fig. 5. Representative load-displacement curve from SRJ indentation of nanocrystalline nickel.

corresponding strain rates and can be clearly distinguished from the baseline strain rate of $2 \times 10^{-2} \mathrm{~s}^{-1}$. The strain rate was kept constant up to $700 \mathrm{~nm}$ to allow that the indentation hardness values to stabilize to a constant value.

Hardness and modulus results as a function of indentation depth are plotted in Fig. 6. The indentation modulus (shown in blue) remains roughly constant as a function of depth, similar to the trends observed by Maier et al. ${ }^{24}$ The scatter in modulus data increases with depth due to the increasing contribution of load cell compliance at higher loads. Because the applied sinus displacement amplitude is not compliance corrected, the compliance-corrected displacement-used for computation of modulus and hardness values-decreases with increasing load. This increases scatter at higher

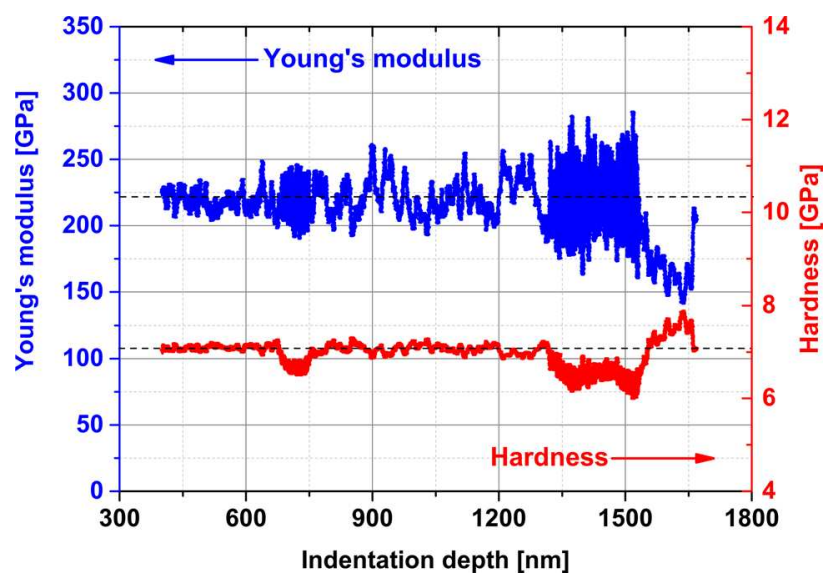

Fig. 6. Indentation modulus (blue) and hardness (red) as a function of indentation depth (Color figure online).

loads and depths. Although not applied here, data smoothing can be additionally used to further reduce the scatter in modulus values. Similar trends have been observed for calibration tests performed on fused silica using this instrument.

Hardness values (shown in red) can be clearly observed to vary directly with the applied strain rate due to the rate jumps. The magnitude of $\Delta H$ is more pronounced for larger $\Delta \varepsilon$ values. Hardness stabilizes back to the baseline values as soon as the initial strain rate is restored. Initial hardness and modulus values from depths less than $400 \mathrm{~nm}$ have not been shown as they are more likely to be affected by tip area calibration and other errors.

\section{Microcompression}

Microcompression is a small-scale characterization technique that allows measurement of small volumes of material comparable with nanoindentation but with a uniaxial stress state. Prior to performing strain-rate jump microcompression tests, constant strain-rate tests were conducted with a strain rate of $\dot{\varepsilon}_{\mathrm{CSR}}=7 \times 10^{-4} \mathrm{~s}^{-1}$ to determine the appropriate strain levels after general yielding to perform rate jumps. Figure 7 illustrates the differences in deformation behavior between the two materials observed in situ during testing at selected strain levels. While the nanocrystalline-nickel micropillar (Fig. 7a) accommodates most of the plastic deformation by somewhat homogeneous barreling localized in its upper half, the singlecrystal pillar (Fig. 7b) develops several large, discrete slip steps across the entire pillar diameter. These slip steps increase in number with increasing strain. However, at higher strain levels, the slip steps near the top of the pillar slide downward and toward the direction of observation to occlude slip steps lower down the pillar.

The stress-strain behavior typical of these CSR tests is shown by the blue curve in Fig. 8 . The yield strength observed at $1 \%$ offset was $2.55 \mathrm{GPa}$. The 


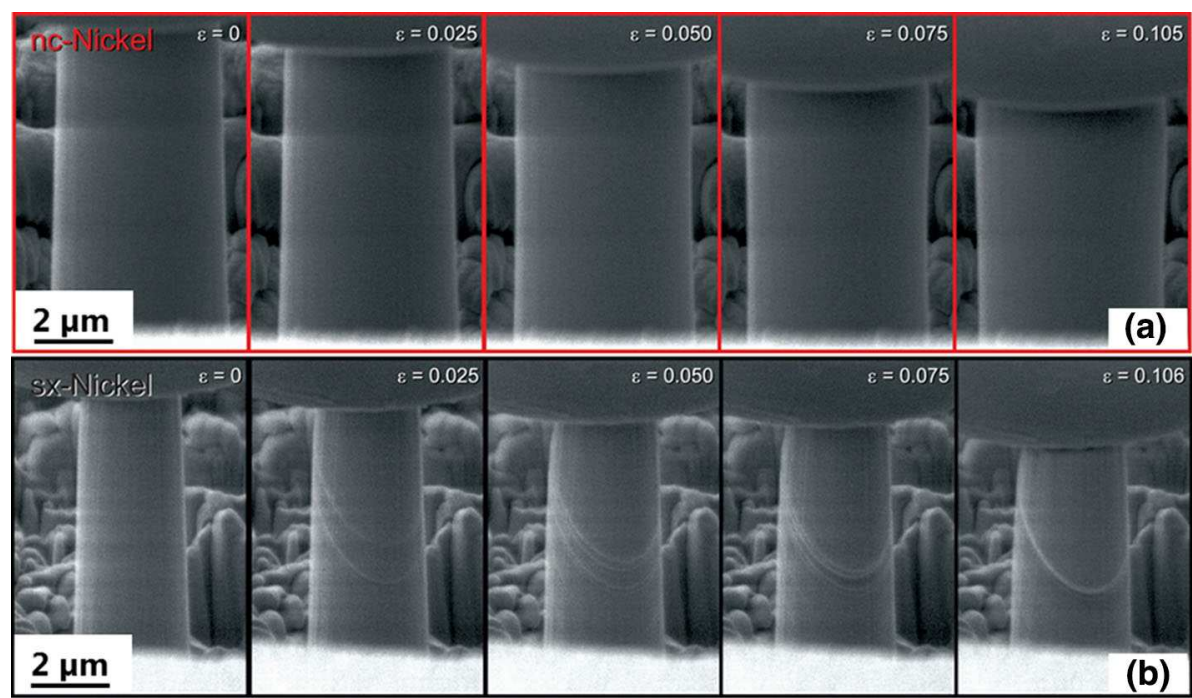

Fig. 7. In situ SEM observation of the deformation behavior of nanocrystalline nickel (a) and single-crystal nickel (b) micropillars during compression testing at selected strains.

apparent strain hardening observed in the curve can be partly attributed to geometric effects and inhomogeneous deformation throughout the pillar height (Fig. 7), as discussed in our previous work. ${ }^{31}$ For all further considerations, only true stressstrain curves were taken into account.

The red curve in Fig. 8 shows the representative true stress-strain behavior observed during the SRJ tests. Strain rates were varied across four orders of magnitude from $4 \times 10^{-5} \mathrm{~s}^{-1}$ to $2 \times$ $10^{-2} \mathrm{~s}^{-1}$ within each test. The jumps are observed to be sharp with rather quick stabilization of the flow stress and minor transients. Following the sequence of the jumps, it is obvious that the increasing strain rates result in a significant increase in flow stress due to the pronounced SRS of nanocrystalline nickel. The first and the final jump segments were both performed at the same strain rate (the same rate as the CSR tests) to allow correction for any influence of apparent work hardening or strain softening. However, the SRJ and CSR tests are shown to agree exceptionally well. This suggests that these tests are highly repeatable due to a uniform microstructure within the material and a high geometric fidelity of the pillars due to their relatively large diameters and careful preparation. Because the flow stress returns to its monotonic and CSR levels after decreasing the strain rate in the final segment, this suggests that any microstructural evolution within the material is effectively independent of strain rate and that no change in deformation mechanism occurs within this range of strain rates.

To illustrate the tremendous strengthening effect of the nanocrystalline microstructure, microcompression testing was also performed on a $\langle 123\rangle$ oriented single crystal of nickel. Both CSR and SRJ

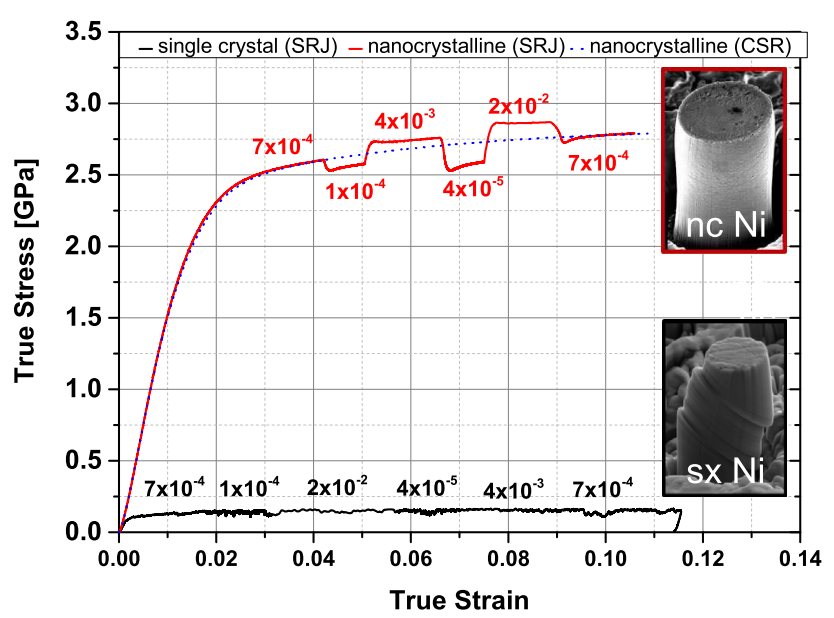

Fig. 8. Micropillar stress-strain curves of nanocrystalline nickel and $\langle 123\rangle$-oriented single-crystal nickel.

tests were conducted on a total of three pillars with exactly the same strain rates and similar diameters as the nanocrystalline micropillars. The single crystal's yield strength at $1 \%$ offset is observed to be only $0.13 \mathrm{GPa}$ (Fig. 8) —nearly a factor of 20 less than its nanocrystalline counterpart! Because of these low strengths, the indenter was operated close to the resolution limit of the load cell, so some additional noise is observed in the data during the rate jumps. However, the deformation of single crystals in experiments similar to this has been frequently observed to display flow serration, which is often attributed to dislocation avalanches $^{54}$ as shown in Fig. 9b. Because of this increased noise, no pronounced strain rate sensitivity can be observed at first glance, and statistical analysis is needed. 


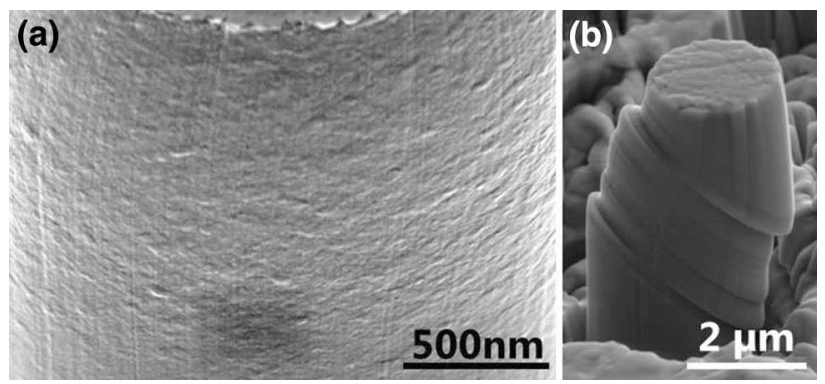

Fig. 9. Postcompression high-resolution scanning electron micrographs illustrate the differences in deformation behavior: (a) nanocrystalline nickel showing displaced grains distributed throughout the pillar and (b) single-crystal nickel showing deformation confined to discrete slip bands traversing the pillar.

\section{ANALYSIS}

\section{Strain-Rate Sensitivity}

For uniaxial mechanical tests (e.g., tension or compression), the SRS exponent $m$ can be extracted by using the following equation: ${ }^{55}$

$$
m=\left[\frac{d(\ln \sigma)}{d(\ln \dot{\varepsilon})}\right]_{T}
$$

where $d(\ln \sigma)$ is the change in flow stress as a function of the change in applied strain rate $d(\ln \dot{\varepsilon})$ at constant temperature $T$. To determine the SRS from indentation tests, the representative indentation strain rate and the representative indentation stress should be used instead. ${ }^{56}$ As in previous work, ${ }^{31}$ two analysis methods were applied to extract values of $m$ from the different data sets. Method 1 allows the direct calculation of the $m$ values from the raw data across the strain-rate jump by ignoring the transients using the assumption that the nanostructure right after the SRJ remains constant. This method is less sensitive to the slope of the curve (strain hardening and softening), but it is susceptible to noise in the data, long transients, and local yield changes immediately before and after the strain rate jump. Method 2 allows determining the SRS from the slope of a plot of stress versus strain rate on a log-log scale, but it is sensitive to any increase or decrease in stress between jumps due to apparent work hardening in the stress-strain curve. It can be seen in both Figs. 4 and 8 that the nanocrystalline nickel does show moderate apparent strain hardening, so additional steps are required to correct for this in order to use method 2. This is accomplished by subtracting the apparent work hardening from the curve by fitting a polynomial relationship to the segments with the same strain rate (i.e., $\dot{\varepsilon}_{\text {SRJ } 1}$ for the curves from tensile tests) and subtracting it from the raw data, as illustrated for the tension tests in Fig. 4. The resulting curves are then flat in regions of constant strain rate so that the variation in flow stress due to changes in strain rate is directly apparent.

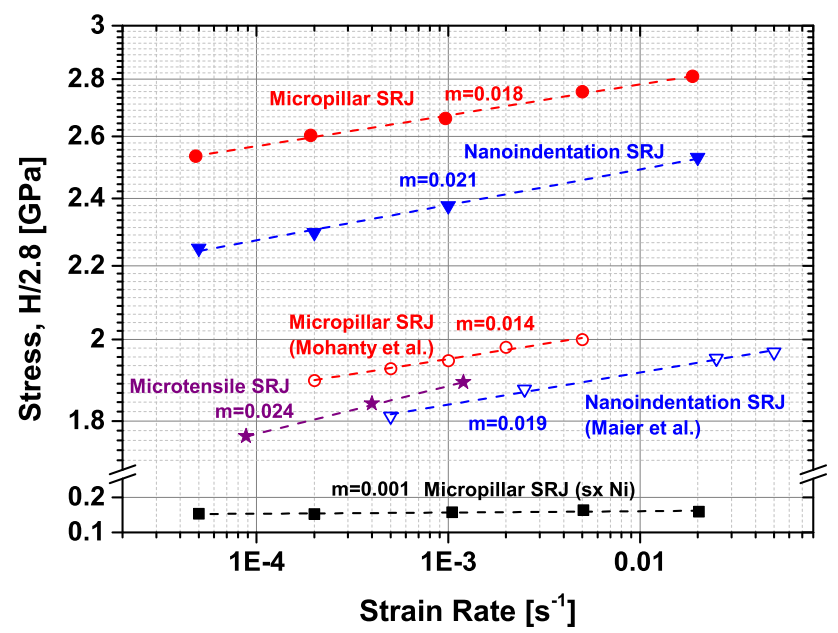

Fig. 10. Flow stress as a function of strain rate for tension, microcompression, and nanoindentation $(H / 2.8)$ tests from the current work and the literature (Maier et al. ${ }^{24}$ and Mohanty et al. ${ }^{31}$ ).

The log-log plot in Fig. 10 compares the resulting yield stress as a function of strain rate for the three applied testing methods. For comparison, the hardness values obtained by nanoindentation were converted into representative stress values by dividing them a confinement parameter of $2.8 .^{24} \mathrm{Al}$ though Fig. 10 compares three different loading cases and three different nanocrystalline-nickel samples with grain sizes of $26 \mathrm{~nm}$ (current study), $27 \mathrm{~nm}$ (Mohanty et al. $^{31}$ ), and $80 \mathrm{~nm}$ (Maier et al. $^{24}$ ), the results are all fairly consistent, especially when comparing the $m$ values between measurements. The variation in terms of absolute strength, rather than relative rate sensitivity, between the small-scale and larger-scale measurements from this study can be attributed to crystallographic texture because XRD measurements revealed a $\langle 111\rangle$ fiber texture in the loading direction of indentation and microcompression experiments. Furthermore, the nanoindentation results are observed to fall between those of the tension and compression tests, which were conducted normal to and parallel to the $\langle 111\rangle$ fiber texture direction, respectively. This is consistent with the triaxial expanding cavity of the indentation plastic zone of a sharp pyramidal tip being less sensitive to anisotropic material properties. ${ }^{57}$

The results from the single-crystal nickel sample, however, vary significantly from the nanocrystalline results in both absolute strength values and SRS exponent values. As mentioned before, the noise in the measurement made extraction of $m$ values difficult, but a value of $m_{\mathrm{sxNi}}=0.001$ was determined by averaging the noise in each jump segment to determine the relative difference. This is one order of magnitude lower than the nanocrystalline nickel results for both rate sensitivity and strength (N.B. the break in scale of the stress-axis for single-crystal-nickel results). 


\section{Apparent Activation Volume}

The strain rate sensitivity exponent measured in the previous section can be used to determine the apparent activation volume of the deformation mechanism using the following relationship: ${ }^{20,25,58}$

$$
m=\frac{\sqrt{3} k T}{V_{\mathrm{app}} \sigma_{\mathrm{f}}} \approx \frac{2.8 \sqrt{3} k T}{V_{\mathrm{app}} H}
$$

where $k$ is the Boltzmann constant, $T$ is the absolute temperature, $V_{\text {app }}$ is the apparent activation volume for plastic deformation, and $\sigma_{\mathrm{f}}$ is the flow stress. It should be noted that an apparent activation volume is used in this context instead of a true or effective activation volume because in strain rate jump tests, it is not possible to infer whether the observed change in flow stress on varying the applied strain rate is due to variation of dislocation density, average dislocation velocity, or both. ${ }^{52}$ Although the concept of activation volume is derived from thermal activation theory of dislocations, it is equally applicable to other nondislocation mechanisms like grain boundary diffusion processes. This is mainly true for nanocrystalline metals in which diffusive mechanisms like grain boundary sliding, grain rotation, and grain boundary migration are active and may predominate. ${ }^{59}$ Furthermore, multiple deformation mechanisms may be active simultaneously, so the apparent volume may not be indicative of a single rate-controlling deformation mechanism.

The room-temperature activation volumes for deformation of nanocrystalline nickel from the current study and the literature ${ }^{16,24,31,60,61}$ are summarized in Table I. The current study reveals nearly identical values of $\sim 10 b^{3}$ for all the different loading cases for the nanocrystalline nickel sample. According to Meyers et al., 59 this corresponds to grain boundary diffusion processes that usually acts in a range from $1 b^{3}$ to $20 b^{3}$. However, it is also within the typical range for dislocation glide-based plasticity, so either or both mechanisms may be active. The literature data summarized in Table I are also in good agreement with the observed values.

Another indication for grain boundary-mediated deformation mechanisms is provided by the surface

Table I. Activation volumes in nanocrystalline nickel

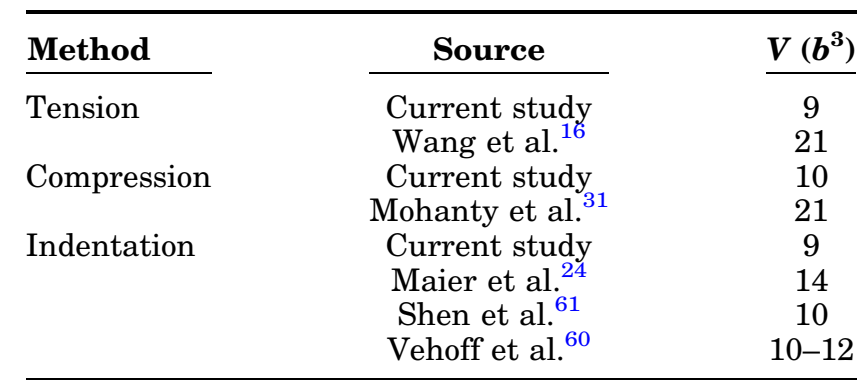

topology of a micropillar after compression. Figure 9a shows evidence of grain rotation or grain boundary sliding of the unconstrained, near-surface grains during the deformation process. This is similar to the relief of surface grains observed during the nanoindentation of ultrafine-grained aluminum $^{25}$ and nanocrystalline nickel. ${ }^{24}$ The deformation observed in the case of the single-crystal nickel (Figs. 8, 9b) is very different than that of the nanocrystalline material. Giant slip offsets are observed across the entire diameter of the micron-scale pillar, rather than the nanoscale protrusions observed in a. It is not too surprising, therefore, that the resulting activation volumes are much larger: $\sim 350 \mathrm{~b}^{3}$. This is consistent with a significant amount of dislocation-dislocation interactions occurring within the pillar but not to the extent of dislocation forest cutting, which would produce volumes $\geq 1000 b^{3}$. ${ }^{62}$ Jennings et al. ${ }^{63}$ observed that for copper pillars with diameters $>0.15 \mu \mathrm{m}$, the rate sensitivity decreases and apparent activation volume increases with increasing pillar diameter. Extrapolating their data to a pillar diameter of $3.5 \mu \mathrm{m}$, the expected activation volume would be $\sim 333 b^{3}$, which is in good agreement with our observed values. This offers further evidence that the applied microcompression methodology for SRS measurements is robust and useful for a wide range of materials and microstructures, even those with very low strain-rate sensitivities.

\section{CONCLUSION}

Three different micromechanical measurement techniques for assessing SRS were applied on the same nanocrystalline nickel specimen to study the comparability and consistency of the test results. The extracted SRS exponents and apparent activation volumes from these three methods were found to be in excellent agreement. This suggests that the rate-controlling deformation mechanism(s) was the same for all three loading cases. The obtained apparent activation volume $\sim 10 b^{3}$ for nanocrystalline nickel is consistent with grain boundary diffusion processes and dislocation glide-based plasticity. Because of the grain size variation in electrodeposited nickel, it is plausible that both of these mechanisms are simultaneously operative. In contrast, the SRS results obtained from microcompression of single-crystal nickel showed much lower SRS and a high apparent activation volume of $\sim 350 \mathrm{~b}^{3}$, which is indicative of significant dislocation-dislocation interactions in the pillars.

This study suggests that the techniques compared herein can be reliably and interchangeably used to measure SRS accurately in a wide variety of materials. However, for materials with mechanical anisotropy, such as crystallographic texture, it is suggested that investigators be aware that nanoindentation techniques underestimate the magnitude of the anisotropy, so alternatives such as tension 
and microcompression are suggested. It is hoped that this investigation will provide the foundation for further studies on other transient testing techniques, like creep and stress relaxation, to understand deformation mechanisms in thin films and nanostructured materials by accurate extraction of deformation parameters.

\section{ACKNOWLEDGEMENTS}

The authors want to thank G. Bürki and A. Böll for technical assistance with the miniature tensile stage and the SEM indenter, and they thank N. Siegfried for performing the GDOES measurements. Funding by the Commission for Technology and Innovation (CTI) within the Project No. 209673 is gratefully acknowledged. G. Mohanty and G. Guillonneau would like to acknowledge funding from the EMPA post-doctoral fellowship program NANOCREEP cofunded by FP7: Marie Curie Actions.

\section{REFERENCES}

1. M.A. Meyers, A. Mishra, and D.J. Benson, Prog. Mater. Sci. 51,427 (2006).

2. L. Wang and B.C. Prorok, J. Mater. Res. 23, 55 (2011).

3. K.S. Kumar, H. Van Swygenhoven, and S. Suresh, Acta Mater. 51, 5743 (2003).

4. G. Palumbo, S.J. Thorpe, and K.T. Aust, Scripta Metall. Mater. 24, 1347 (1990).

5. N. Wang, Z. Wang, K.T. Aust, and U. Erb, Acta Metall. Mater. 43, 519 (1995).

6. N.Q. Chinh, P. Szommer, Z. Horita, and T.G. Langdon, Adv. Mater. 18, 34 (2006).

7. H. Van Swygenhoven, Mater. Sci. Eng. A 483-484, 33 (2008).

8. R.L. Coble, J. Appl. Phys. 34, 1679 (1963).

9. T. Langdon, J. Mater. Sci. 41, 597 (2006).

10. R. Valiev, Nat. Mater. 3, 511 (2004).

11. F. Dalla Torre, H. Van Swygenhoven, and M. Victoria, Acta Mater. 50, 3957 (2002).

12. Y.F. Shen, L. Lu, Q.H. Lu, Z.H. Jin, and K. Lu, Scripta Mater. 52, 989 (2005).

13. E. Ma, Y.M. Wang, Q.H. Lu, M.L. Sui, L. Lu, and K. Lu, Appl. Phys. Lett. 85, 4932 (2004).

14. Y.M. Wang and E. Ma, Mater. Sci. Eng. A 375-377, 46 (2004).

15. J.-Y. Kim and J.R. Greer, Acta Mater. 57, 5245 (2009).

16. Y. Wang, A. Hamza, and E. Ma, Acta Mater. 54, 2715 (2006).

17. N. Karanjgaokar, K. Jonnalagadda, I. Chasiotis, J. Chee, A. Mahmood, and D. Peroulis, Paper presented at the Proceedings XIth International Congress and Exposition (Orlando, FL, 2008).

18. K.N. Jonnalagadda, I. Chasiotis, S. Yagnamurthy, J. Lambros, J. Pulskamp, R. Polcawich, and M. Dubey, Exp. Mech. 50, 25 (2009).

19. D.S. Gianola, D.H. Warner, J.F. Molinari, and K.J. Hemker, Scripta Mater. 55, 649 (2006).

20. Q. Wei, S. Cheng, K. Ramesh, and E. Ma, Mater. Sci. Eng. A 381,71 (2004).

21. M.J. Mayo and W.D. Nix, Acta Metall. 36, 2183 (1988).

22. R. Schwaiger, B. Moser, M. Dao, N. Chollacoop, and S. Suresh, Acta Mater. 51, 5159 (2003).

23. D. Pan and M.W. Chen, J. Mater. Res. 24, 1466 (2011).

24. V. Maier, K. Durst, and J. Mueller, J. Mater. Res. 28, 1421 (2011).
25. J.M. Wheeler, V. Maier, K. Durst, M. Göken, and J. Michler, Mater. Sci. Eng. A 585, 108 (2013).

26. V. Maier, C. Schunk, M. Göken, and K. Durst, Philos. Mag. 1-14 (2014).

27. M. Uchic, D. Dimiduk, J. Florando, and W. Nix, Science 305, 986 (2004).

28. D. Kiener, W. Grosinger, G. Dehm, and R. Pippan, Acta Mater. 56, 580 (2008).

29. J.M. Wheeler, C. Niederberger, C. Tessarek, S. Christiansen, and J. Michler, Int. J. Plast. 40, 140 (2013).

30. J.S. Carpenter, A. Misra, M.D. Uchic, and P.M. Anderson, Appl. Phys. Lett. 101, 051901 (2012).

31. G. Mohanty, J.M. Wheeler, R. Raghavan, J. Wehrs, M. Hasegawa, S. Mischler, L. Philippe, and J. Michler, Philos. Mag. 1-18 (2014).

32. V. Saile, LIGA Appl. 7, 1 (2009).

33. T.J. Rupert, J.C. Trenkle, and C.A. Schuh, Acta Mater. 59 , 1619 (2011).

34. J.R. Trelewicz and C.A. Schuh, Acta Mater. 55, 5948 (2007).

35. A. Detor and C. Schuh, Acta Mater. 55, 371 (2007).

36. W.M. Yin, S.H. Whang, R. Mirshams, and C.H. Xiao, Mater. Sci. Eng. A 301, 18 (2001).

37. W.M. Yin and S.H. Whang, Scripta Mater. 44, 569 (2001).

38. W.M. Yin, S.H. Whang, and R.A. Mirshams, Acta Mater. 53, 383 (2005).

39. Y.M. Wang, S. Cheng, Q.M. Wei, E. Ma, T.G. Nieh, and A. Hamza, Scripta Mater. 51, 1023 (2004).

40. C.S. Barret and T.B. Massalski, Structure of Metals (Oxford: Pergamon Press, 1980).

41. P. Scherrer, Nachrichten von Der Gesellschaft Der Wissenschaften 98-100 (1918).

42. J. Langford and A. Wilson, J. Appl. Crystallogr. 11, 102-113 (1978)

43. L.A. Giannuzzi and F.A. Stevie, Micron 30, 197 (1999).

44. L. Philippe, P. Schwaller, G. Bürki, and J. Michler, J. Mater. Res. 23, 1383 (2011).

45. B. Lucas and T. Kanade, IJCAI 121-130 (1981).

46. R. Rabe, J.M. Breguet, P. Schwaller, S. Stauss, F.J. Haug, and J. Patscheider, Thin Solid Films 469-470, 206 (2004).

47. J.M. Wheeler, P. Brodard, and J. Michler, Philos. Mag. 92, 3128 (2012).

48. W.C. Oliver and G.M. Pharr, J. Mater. Res. 7, 1564 (1992).

49. S.A.S. Asif, K.J. Wahl, and R.J. Colton, Rev. Sci. Instrum. 70, 2408 (1999).

50. W. Oliver and G. Pharr, J. Mater. Res. 19, 3 (2004).

51. B.N. Lucas and W.C. Oliver, Metall. Mater. Trans. A 30, 601 (1999).

52. D. Caillard and J.L. Martin, Pergamon Mater. Ser. 8, 3 (2003).

53. G.E. Dieter and D. Bacon, Mechanical Metallurgy (New York: McGraw-Hill, 1986), p. 751.

54. F.F. Csikor, C. Motz, D. Weygand, M. Zaiser, and S. Zapperi, Science 318, 251 (2007).

55. E. Hart, Acta Metall. 15, 351 (1967).

56. R. Goodall and T.W. Clyne, Acta Mater. 54, 5489 (2006).

57. J.J. Vlassak and W.D. Nix, Philos. Mag. A 67, 1045 (1993).

58. L. Lu, R. Schwaiger, Z.W. Shan, M. Dao, K. Lu, and S. Suresh, Acta Mater. 53, 2169 (2005).

59. M.A. Meyers, A. Mishra, and D.J. Benson, Prog. Mater. Sci. 51,427 (2006).

60. H. Vehoff, D. Lemaire, K. Schüler, T. Waschkies, and B. Yang, Int. J. Mater. Res. 98, 259 (2007).

61. X. Shen, J. Lian, Z. Jiang, and Q. Jiang, Mater. Sci. Eng. A 487, 410 (2008).

62. A.G. Evans and R.D. Rawlings, Phys. Status Solidi 34, 9 (1969).

63. A.T. Jennings, J. Li, and J.R. Greer, Acta Mater. 59, 5627 (2011). 